\title{
Malaria and intestinal parasite co-infections and impact on haematological parameters among people living with HIV in Buea, Southwest Cameroon
}

\author{
Sorelle Mekachie Sandie ( $\square$ sandiesorelle2@gmail.com ) \\ University of Buea https://orcid.org/0000-0003-3396-0983 \\ Irene Ule Ngole Sumbele \\ Cornell University College of Veterinary Medicine \\ Martin Mih Tasah \\ University of Buea \\ Helen Kuokuo Kimbi \\ University of Bamenda
}

\section{Research Article}

Keywords: Anaemia, co-infection, intestinal parasites, malaria parasite, haematological parameters, PLWH, Cameroon

Posted Date: April 15th, 2020

DOI: https://doi.org/10.21203/rs.3.rs-22401/v1

License: (c) (i) This work is licensed under a Creative Commons Attribution 4.0 International License. Read Full License 


\section{Abstract}

Background: Both malaria and intestinal parasites are endemic in Cameroon, and their co-infection can be of great impact on haematological parameters among people living with HIV (PLWH). This crosssectional study determined the prevalence and impact of malaria/intestinal parasite co-infections on haematological parameters in PLWH and HIV negative individuals in Buea, Cameroon from March-August 2019.

Methods: The study population (500) comprised of 190 PLWH, 216 HIV consenting negative individuals from the Buea community and 94 HIV negative patients at the Buea Hospital out-patient department (OPD). Participants were examined clinically, and the collected blood sample was used for malaria parasite (MP) detection, HIV diagnosis and full blood count analysis. Stool samples were examined for the detection of intestinal parasites (IPs) using the formol-ether concentration and modified Zheil-Neelsen techniques. Proportions were compared using Pearson's Chi-square test and association of anaemia with the independent variables was evaluated using multivariate logistic regression analysis

Results: Overall, MP, IPs and MP/IPs co-infection prevalences were 17.0\% (85), 13.0\% (65) and 2.2\% (11) respectively. When compared with HIV negative participants from the communities, PLWH had the highest prevalence of MP (16.3\%, P = 0.17), IPs (23.7\%, $\mathrm{P} \otimes 0.001)$ and MP/IPs co-infection $(3.7 \%, \mathrm{P}=$ 0.04). Plasmodium falciparum was the only malaria parasite identified. Detected protozoa $(10.2 \%)$ were Cryptosporidium species and Entamoeba histolytica while detected helminths (3.8\%) were Ascaris lumbricoides, Schistosoma mansoni, hookworm, Dipylidium caninum, Hymenolepis nana and Taenia species. Cryptosporidium species $(16.8 \%, 32)$ was the most prevalent protozoan IP while hookworm $(2.6 \%, 5)$ was the most prevalent helminth among PLWH. The significant risk factors associated with anaemia included being aged $21-30$ years $(P=0.029)$, a male $(P<0.001)$, MP positive $(P<0.001), H I V$ positive $(P<0.001)$ and feverish $(P<0.001)$. Participants co-infected with HIV, MP and IP had a significantly lower mean haemoglobin value $(10.56 \pm 1.21 \mathrm{~g} / \mathrm{dl})$.

Conclusion: Malaria and intestinal parasites are still public health concern among PLWH and coinfections have negative influence on haematological parameters especially haemoglobin concentration. Routine screening for malaria and IPs in PLWH is recommended, as well as haemoglobin concentration monitoring.

\section{Background}

Parasitic infections are a major cause of morbidity and mortality in Africa; especially in resource limited tropical and sub-tropical regions like sub-Saharan Africa [1] where the majority of HIV/AIDS cases are concentrated. People living with HIV (PLWH) are threatened by a great number of diseases caused by different types of biological agents.

The number of new HIV infections globally continued to decline in 2017, from 3.4 million cases in 1996 to 1.8 million cases in 2017 [2]. HIV infection is characterized by immunosuppression that exposes the 
infected individual to a variety of microbial and parasitic infections such as Plasmodium, Mycobacterium tuberculosis and intestinal parasitic infections. These infections (opportunistic or not) are a serious threat to the health status of people living with HIV, especially in developing countries like Cameroon. To achieve HIV epidemic control in 2021, the Cameroon government has been implementing various strategies that led to an encouraging increase in anti-retroviral therapy (ART) coverage (53\%) and viral load (VL) suppression (44.7\%) nationally in 2019 [3].

WHO African region still bears the largest burden of malaria morbidity (93\%), with Plasmodium falciparum accounting for $99.7 \%$ of malaria cases in 2018 [4]. WHO reported Cameroon as the fourth Sub-Saharan African country behind Nigeria, Democratic Republic of Congo and Mozambique responsible for the malaria cases in the region [5]. Many studies have consistently reported an association between HIV infection and malaria in Cameroon $[6,7,8,9,10]$ In Cameroon, each region shows varied prevalence of both malaria and HIV infection. HIV prevalence is approximately $3.7 \%$ [3] while malaria transmission is stable throughout the year, with an overall malaria test positivity rate of $62 \%$ registered in 2017 [11].

The burden of intestinal parasite infections (IPIs) is greatest in low-income areas and are major public health problems in certain areas of Sub-Saharan Africa. These IPIs are associated with humid climate, unsanitary environments and poor socio-economic conditions. Parasites causing these IPIs are protozoa (Cryptosporidium species, Cyclospora cayetanensis, Isospora belli, and Microsporidia spp, Entamoeba histolytica) and helminthes (Ascaris lumbricoides, Trichuris trichuiria, hookworms, Schistosoma mansoni, Taenia species). Cryptosporidium species, Cyclospora cayetanensis, Isospora belli, and Microsporidia spp are often referred to as opportunistic parasites in people living with HIV and cause great morbidity when CD4 T cell count falls below 200 cells/ $\mu$ [12]. Various studies carried out among African adults living with HIV have reported IPI rates ranging from $10-45 \%[13,14]$. Majority of the research on the impact of IPIs on HIV progression in Cameroon, occurred before the wide availability of ART [15] hence, the need for further evaluation.

In Cameroon, PLWH face similar environmental realities as those not infected with HIV and so can easily get co-infected with combinations of intestinal and malaria parasites. Such co-infections may have considerable health consequences which could lead to severe clinical symptoms and pathology in PLWH. Studies have shown that individuals co-infected with more than one parasite species have an increased risk of developing more frequent and severe diseases due to interactions among infecting parasite species $[16,17,18]$.

Malaria and intestinal parasites in PLWH can both lead to severe haematological abnormalities. Although, ART in combination with cotrimoxazole confers a level of immunity to infections in PLWH, it has been reported to have adverse effects on the red blood cell indices of these patients, thereby causing anaemia [19]. Although malaria and intestinal parasites as well as ART are known etiological factors in anaemia $[20,21,15,10]$, the extent to which their interaction might enhance the risk of the haematological abnormalities merits further investigations. Previous studies have reported on the 
influence of malaria/HIV co-infection on the occurrence of anaemia $[22,18,10]$. The situation regarding PLWH co-infected with malaria and intestinal parasites in the area following the intensification of different control strategies such free availability of ART and cotrimoxazole, access to viral load testing and community ART dispensation is unknown. Therefore, the present study was undertaken to determine the prevalence of malaria parasite and intestinal parasites in PLWH compared to HIV negative counterparts as well as to assess the effects of these co-infections on clinical and haematological parameters in order to provide relevant information to policy makers in the country.

\section{Methods}

\section{Study area and participants}

Participants were enrolled from the Buea Regional Hospital (HIV Treatment centre and Out-patient unit) and from different neighbourhoods in Buea. Buea has a population of about 200,000 inhabitants and it is the South West Regional capital. The Buea Regional hospital is the referral HIV treatment centre in Fako Division serving the population. With the presence of the Mount Cameroon, Buea is a major touristic site and has a business-friendly environment. Its inhabitants are from almost all ethnic groups of Cameroon. In the national HIV prevalence ranking, the South West Region is the 6th Region with a prevalence of 3.6\% which equates the national prevalence (3.7\%) [23] as well as the second to the last Region (33.8\%) with the least viral load suppression. Buea is an area of moderate malaria transmission, with peak time for malaria transmission during and just after the rainy season (March to September) [24]. Its tropical climate provides a suitable environment for the development of the malaria vectors and this therefore contributes to increased malaria transmission. There is also inadequate access to safe water in this region, predisposing the population to water-borne infections including IPIs.

Three categories of participants; PLWH at the HIV Treatment centre, HIV-negative individuals on consultation at the outpatient department (OPD) and HIV-negative individuals in different neighbourhood were enrolled in the study. Participants were recruited irrespective of their age, sex, marital status, occupation, level of education; with or without malaria-related and intestinal parasite infections-related signs and symptoms, ART regimen and duration on ART. Only participants who signed the informed consent form and willingly accepted to be counselled and tested for malaria, IPIs and HIV were enrolled in the study. Participants on antibiotics, malaria treatment or antiparasitic agents two weeks prior to the sample collection were excluded from the study.

\section{Study Design And Sample Size}

This study was a hospital and community-based cross-sectional study carried out within April 2018 February 2019. HIV-negative participants included persons who consented to be screened for HIV during the process and were negative while apparently healthy HIV-negative individuals were those enrolled through HIV outreach programs in their homes and offices in the Buea neighbourhoods (Molyko, 
Bonduma, Great Soppo, GRA, Buea town, Bokwaongo, Bokova, Mile 18, Clerks and Federal quarters). The sample size was determined using the formula $n=Z^{2} p q / d^{2}[25]$ where $n$ represented the sample size evaluated, $Z$ was 1.96 , which is the standard normal deviate (for the $95 \%$ confidence interval, $\mathrm{Cl}$ ), $\mathrm{p}$ was $14.1 \%$ [10] and 82.6\% [15], the prevalence of malaria and IPIs in PLWH and HIV negative participants respectively, q was $1-p$ and $d$ was 0.05 . The sample size calculated for malaria parasite prevalence was 186 while that for IPIs prevalence was 220 given an optimum sample size for the study of 406 participants.

\section{Data Collection}

\section{Questionnaire}

A pre-structured questionnaire was used to collect socio-demographic information such as age, sex, location of residence, level of education, marital status, occupation while clinical data such as ART usage, duration on treatment, ART regimen, viral load (VL) were collected from each HIV patient's file at the HIV centre. Health and preventive practices towards malaria and IPIs as well as behavioural habits regarding health conditions with a history of symptoms were recorded. Environmental conditions such as the water supply, swimming practices and animal ownership were also recorded for all the patients.

\section{Sample Collection And Processing}

Participants were instructed on how to collect stool sample in universal transparent faecal containers with a screw cap lid. The samples were transported to the Malaria Research Laboratory of the University of Buea. On arrival in the laboratory, the stool samples were analysed macroscopically and preserved in $10 \%$ formalin for 24 hours for further analysis. Formol-ether concentration technique and modified ZiehlNeelsen (ZN) method [26] were performed for the detection of intestinal parasites (protozoa and helminths).

After provision of a stool sample, the participant's body temperature was recorded using a digital thermometer and fever was defined as temperature $\geq 37.5^{\circ} \mathrm{C}$. Blood was collected under sterile conditions into two well-labelled Ethylenediaminetetraacetate (EDTA) tubes for each participant for HIV screening and confirmation, malaria diagnosis and full blood count (FBC). Part of the blood was used to prepare thick and thin blood films on a labelled slide that was Giemsa-stained and analysed following standard procedures [26] by two experienced microscopists. The blood in one of the tubes was used for HIV rapid test (in the case of presumed HIV negative participants), CD4 T-cell count and to conduct FBC. Viral loads were obtained from the HIV patient's file at the HIV Care Centre. Viral load $\otimes 50$ copies $/ \mathrm{mL}$ of blood was considered undetectable [27].

\section{Haematological Analysis}


The FBC analysis was carried out using the URIT-3300 automated haematology analyser (URIT Medical Electronic CO., LTD. Jiuhua Road, Gungxi, China), following the manufacturer's instructions. Values of the following parameters were obtained from the haematology analyser: White blood cell (WBC), red blood cell (RBC) and platelet counts, haemoglobin concentration ( $\mathrm{Hb}$ ), haematocrit ( $\mathrm{Hct})$, mean corpuscular volume (MCV), mean corpuscular haemoglobin $(\mathrm{MCH})$, mean corpuscular haemoglobin concentration $(\mathrm{MCHC})$. Anaemia was defined as haemoglobin concentrations below the World Health Organization (WHO) reference values corresponding to age or gender [28]. Leucopenia was defined as WBC $<4000$ cells/ $\mu \mathrm{L}$, microcytosis as MCV less than $80 \mathrm{fL}$ [29] and thrombocytopenia was defined as platelet count < 150000 cells/ $\mu \mathrm{L}$. CD4T cell counts were determined using the BD FACS Count ${ }^{\text {TM }}$ System (BD Biosciences, USA) as per the manufacturer's instructions. To assure the quality of the laboratory data, standard operating procedure for each test was followed. Reagents were checked for expiry date and prepared according to the manufacturer's instruction. The Determine Rapid Test Kit (Abott Laboratories, CO., Ltd. Minato-Ku, Tokyo Japan) was used to test the supposed HIV negative subjects for HIV-1 following the manufacturer's instruction.

\section{Statistical Analysis}

Data was analysed using IBM-Statistical Package for the Social Science (SPSS) version 20.0 software (SPSS Inc., Illinois, USA). Baseline characteristics was summarized as mean with $95 \% \mathrm{Cl}$ and compared using the independent $t$-test. Malaria and intestinal parasites data were summarized into means and standard deviation (SD) and percentages were used in the evaluation of the descriptive statistics. The significance of differences in prevalence was explored using Pearson's Chi-square while Analysis of Variance (ANOVA) or Student's t test were used where appropriate to assess difference in group means. The association of anaemia with the independent variables was evaluated using multivariate logistic regression analysis. $P<0.05$ was considered for statistical significance.

\section{Ethical Considerations}

Using an information sheet, a brief talk was given to the participants in English language (exceptionally in Pidgin English) and the participants were then invited to participate in the study by signing an informed consent form. In the case of children ( $\leq 15$ years), their parents signed proxy informed consent form on their behalf. Ethical clearance was obtained from the Institutional Review Board hosted by the Faculty of Health Sciences of the University of Buea (Reference Number: 661-09). For confidentiality purposes, patients were identified by special identification numbers. Results of participants with parasitic infections in the neighbourhoods were sent as soon as possible to a clinician for their case management.

\section{Results}

\section{Socio-demographic and clinical characteristics of the participants}


Six hundred and eighty three (683) participants were approached to participate in the study, with 500 (73.2\%) being successfully enrolled. Out of the 500 participants, 190 were HIV positive patients from the HIV Treatment Centre, 216 were HIV negative from the communities and 94 were HIV negative from the OPD of the Buea Regional Hospital as shown in Fig. 1.

Overall, the mean (SD) age of the participants was 30.0 (17.1) years. There were more females $(66.0 \%)$ than males (34.0\%). A greater proportion of the study population belonged to the $\leq 20$ years age group (31.6\%) and the least to the 31-40 years age group (17.6\%) as shown in Table 1. Majority of the PLWH had primary level of education (66.3\%), were self-employed (31.2\%) and were single (62.0\%). Among HIV negative participants (in communities and at OPD respectively), majority were students $(56.5 \%, 56.5 \%)$ and were single $(63.0 \%, 51.0 \%)$ as revealed in Table 1. 
Table 1

Socio-demographic profile of the study population

\begin{tabular}{|c|c|c|c|c|}
\hline \multirow[t]{2}{*}{ Parameters } & HIV Positive & Communities HIV Negative & $\begin{array}{l}\text { OPD } \\
\text { HIV Negative }\end{array}$ & Overall \\
\hline & n (\%) & n (\%) & $\mathrm{n}(\%)$ & $\mathrm{n}(\%)$ \\
\hline $\begin{array}{l}\text { Age groups (years) } \\
\leq 20\end{array}$ & $42(22.1)$ & $72(33.3)$ & $44(46.8)$ & $158(31.6)$ \\
\hline $21-30$ & $20(10.5)$ & $70(32.4)$ & $22(23.4)$ & $112(22.4)$ \\
\hline $31-40$ & $48(25.3)$ & $28(13.0)$ & $12(12.8)$ & $88(17.6)$ \\
\hline$>40$ & $80(42.1)$ & $46(21.3)$ & $16(17.0)$ & $142(28.4)$ \\
\hline Mean \pm SD age & $35.5 \pm 16.5$ & $27.6 \pm 15.8$ & $24.0 \pm 17.9$ & $30.0 \pm 17.1$ \\
\hline \multicolumn{5}{|l|}{ Sex } \\
\hline Female & $140(73.7)$ & $138(63.9)$ & $52(55.3)$ & $330(66.0)$ \\
\hline Male & $50(26.3)$ & $78(36.1)$ & $42(44.7)$ & $170(34.0)$ \\
\hline \multicolumn{5}{|l|}{ Educational Level } \\
\hline No formal & $7(3.7)$ & $18(8.6)$ & $22(23.9)$ & $47(9.5)$ \\
\hline Primary & $126(66.3)$ & $54(25.7)$ & $28(30.4)$ & $208(42.3)$ \\
\hline Secondary & $43(22.6)$ & $64(30.5)$ & $18(19.6)$ & $125(25.4)$ \\
\hline Tertiary & $14(7.4)$ & $74(35.2)$ & $24(26.1)$ & $112(22.8)$ \\
\hline \multicolumn{5}{|l|}{ Occupation } \\
\hline Student & $48(25.4)$ & $122(56.5)$ & $52(56.5)$ & $222(44.7)$ \\
\hline Unemployed & $25(13.2)$ & $12(5.2)$ & $12(13.0)$ & $49(9.9)$ \\
\hline Self-employed & $59(31.2)$ & $28(13.0)$ & $8(8.7)$ & $95(19.1)$ \\
\hline Employee & $57(30.2)$ & $54(25.0)$ & $20(21.7)$ & $131(26.4)$ \\
\hline \multicolumn{5}{|l|}{ Marital status } \\
\hline Single & $93(62.0)$ & $102(63.0)$ & $25(51.0)$ & $220(60.9)$ \\
\hline Married & $61(38.0)$ & $60(37.0)$ & $24(49.0)$ & 141(39.1) \\
\hline ITN usage & $109(57.4)$ & 76 (35.2) & 38 (40.4) & $223(44.6)$ \\
\hline
\end{tabular}

Axillary temperature measurement revealed $15.0 \%$ of the general population were feverish, with the highest proportion (19.1\%) among HIV negative patients at the OPD. Clinical signs (headache, joint pains, 
nausea, vomiting etc...) were less frequent among HIV negative participants from the communities than among HIV negatives at the OPD (40.5\%). Anaemia occurred in $56.6 \%$ (283) of the participants and PLWH had the highest occurrence (76.8\%) when compared with their HIV negative counterparts. Among PLWH, half of the population (50.0\%) had CD4 T cell count between $200-500$ cells/ $\mu \mathrm{L}$ of blood, majority (97.9\%) were on ART, 91.9\% (171) were taking first line ART regimen and $77.5 \%$ (141) had attained viral suppression( $\mathbb{\square} 50$ copies $/ \mathrm{mL}$ of blood) as shown in Table 2 . 
Table 2

Clinical characteristics and laboratory profile of the study population

\begin{tabular}{|c|c|c|c|c|}
\hline Parameters & $\begin{array}{l}\text { HIV } \\
\text { Positive } \\
\text { n (\%) }\end{array}$ & $\begin{array}{l}\text { Communities HIV } \\
\text { Negative } \\
\mathrm{n}(\%)\end{array}$ & $\begin{array}{l}\text { OPD } \\
\text { HIV } \\
\text { Negative } \\
\text { n (\%) }\end{array}$ & $\begin{array}{l}\text { Overall } \\
\mathrm{n}(\%)\end{array}$ \\
\hline $\begin{array}{l}\text { Fever status } \\
\text { Febrile }\end{array}$ & $23(12.1)$ & $34(15.7)$ & $18(19.1)$ & $75(15.0)$ \\
\hline Afebrile & 167 (87.9) & $182(84.3)$ & $76(80.9)$ & $425(85.0)$ \\
\hline Mean temperature (SD) in ${ }^{\circ} \mathrm{C}$ & $37.1(0.5)$ & $37.1(0.5)$ & $37.3(0.5)$ & $37.1(0.5)$ \\
\hline $\begin{array}{l}\text { Presence of clinical signs } \\
\text { Yes }\end{array}$ & $39(23.5)$ & $32(18.2)$ & $30(40.5)$ & $101(24.3)$ \\
\hline No & $127(76.5)$ & $144(81.8)$ & $44(59.5)$ & $315(75.7)$ \\
\hline $\begin{array}{l}\text { Anaemic status } \\
\text { Anaemic }\end{array}$ & $146(76.8)$ & $86(39.8)$ & $51(54.3)$ & $283(56.6)$ \\
\hline Non-anaemic & $44(23.2)$ & $130(60.2)$ & $43(45.7)$ & $217(43.4)$ \\
\hline Mean $\mathrm{Hb}(\mathrm{SD})$ in $\mathrm{g} / \mathrm{dL}$ & $11.1(1.5)$ & $12.5(2.1)$ & $12.1(2.8)$ & $11.9(2.2)$ \\
\hline $\begin{array}{l}\text { CD4 T-cell count(cells } / \mu \mathrm{L}) \\
<200\end{array}$ & $13(12.5)$ & - & - & $13(12.5)$ \\
\hline $200-500$ & $52(50.0)$ & - & - & $52(50.0)$ \\
\hline$>500$ & $39(37.5)$ & - & - & $39(37.5)$ \\
\hline $\begin{array}{l}\text { Mean CD4 T cell count SD } \\
\text { (cells } / \mu \mathrm{L})\end{array}$ & $\begin{array}{l}466.0 \\
(275.9)\end{array}$ & - & - & $\begin{array}{l}466.0 \\
(275.9)\end{array}$ \\
\hline $\begin{array}{l}\text { ART usage } \\
\text { Yes }\end{array}$ & $186(97.9)$ & - & - & $186(97.9)$ \\
\hline No & $4(2.1)$ & - & - & $4(2.1)$ \\
\hline $\begin{array}{l}\text { ART regimen } \\
\text { First line }\end{array}$ & 171 (91.9) & - & - & 171 (91.9) \\
\hline Second line & $15(8.1)$ & - & - & $15(8.1)$ \\
\hline $\begin{array}{l}\text { ART Duration (years) } \\
<1\end{array}$ & $36(19.4)$ & - & - & $36(19.4)$ \\
\hline $1-3$ & $61(32.8)$ & - & - & $61(32.8)$ \\
\hline$>3$ & $89(47.8)$ & - & - & $89(47.8)$ \\
\hline
\end{tabular}




\begin{tabular}{|c|c|c|c|c|}
\hline Parameters & $\begin{array}{l}\text { HIV } \\
\text { Positive } \\
\text { n (\%) }\end{array}$ & $\begin{array}{l}\text { Communities HIV } \\
\text { Negative } \\
\mathrm{n}(\%)\end{array}$ & $\begin{array}{l}\text { OPD } \\
\text { HIV } \\
\text { Negative } \\
\mathrm{n}(\%)\end{array}$ & $\begin{array}{l}\text { Overall } \\
\text { n (\%) }\end{array}$ \\
\hline $\begin{array}{l}\text { Viral load } \\
\text { (RNA copies/ml) }\end{array}$ & & - & - & \\
\hline Not detected $(\varangle 50)$ & $141(77.5)$ & - & - & $141(77.5)$ \\
\hline$\geq 50$ & $41(22.5)$ & - & - & $41(22.5)$ \\
\hline
\end{tabular}

\section{Malaria Parasite Prevalence}

Out of the 500 participants, 85 (17.0\%) were positive for MP with Plasmodium falciparum as the only species observed. Although not statistically significant $\left(\chi^{2}=3.52 \mathrm{P}=0.17\right)$, MP prevalence was higher in HIV negatives at the OPD (23.4\%) and least in HIV negatives in the communities (14.8\%) as shown in Fig. 2.

With respect to gender and age MP prevalence was significantly higher in HIV negative females in the OPD $(30.8 \%)$ and the HIV negatives $>40$ years in the communities $(26.1 \%)$ than their respective counterparts. Overall, the prevalence of MP was significantly higher in those febrile (85.3\%) and those anaemic (23.0\%) than their respective counterparts. Specifically, significantly higher prevalence of MP was observed in those febrile among PLWH (87.0\%), HIV negatives in the communities $(82.4 \%)$ and HIV negatives in the OPD (88.9\%). Similar trend in significantly higher prevalence of MP was observed in those anaemic among HIV negative participants in the communities and the OPD (P凶0.001, P $\otimes 0.001)$ as shown in Table 3. With respect to the presence of clinical signs, malaria parasite was most prevalent in HIV negative participants presenting themselves at the OPD (33.3\%) unlike their contemporaries $\left(\chi^{2}=\right.$ $4.09, P=0.04)$. Those who attained viral suppression and those with CD4 T-cell count between 200-500 cells// $\mu \mathrm{l}$ had the highest prevalence of MP $(17.0 \%$ and $21.6 \%$ respectively) although the difference was not statistically significant (Table 3 ). 
Table 3

Malaria parasite prevalence among the different categories of patients with respect to socio-demographic and clinical data

\begin{tabular}{|c|c|c|c|c|c|c|c|c|}
\hline \multirow[t]{2}{*}{ Categories } & \multicolumn{2}{|c|}{$\begin{array}{l}\text { HIV } \\
\text { Positive }\end{array}$} & \multicolumn{2}{|c|}{$\begin{array}{l}\text { Community HIV } \\
\text { Negative }\end{array}$} & \multicolumn{2}{|c|}{$\begin{array}{l}\text { OPD } \\
\text { HIV Negative }\end{array}$} & \multicolumn{2}{|c|}{ Overall } \\
\hline & $\mathbf{N}$ & $\begin{array}{l}\text { Malaria } \\
\text { parasite } \\
\text { positive \% (n) }\end{array}$ & $\mathbf{N}$ & $\begin{array}{l}\text { Malaria } \\
\text { parasite } \\
\text { positive \% } \\
\text { (n) }\end{array}$ & $\mathbf{N}$ & $\begin{array}{l}\text { Malaria } \\
\text { parasite } \\
\text { positive \% } \\
\text { (n) }\end{array}$ & $\mathbf{N}$ & $\begin{array}{l}\text { Malaria } \\
\text { parasite } \\
\text { positive } \\
\%(n)\end{array}$ \\
\hline $\begin{array}{l}\text { Gender } \\
\text { Male }\end{array}$ & 50 & $18.0(9)$ & 78 & $15.4(12)$ & 42 & $9.5(4)$ & 170 & $\begin{array}{l}14.7 \\
(25)\end{array}$ \\
\hline Female & 140 & $15.7(22)$ & 138 & $14.5(20)$ & 52 & $30.8(16)$ & 330 & $\begin{array}{l}17.6 \\
(58)\end{array}$ \\
\hline $\begin{array}{l}\text { Level of } \\
\text { Significance }\end{array}$ & \multicolumn{2}{|c|}{$\chi^{2}=0.18, P=0.41$} & \multicolumn{2}{|c|}{$\chi^{2}=0.31, P=0.50$} & \multicolumn{2}{|c|}{$\begin{array}{l}\chi^{2}=6.26, P= \\
0.01^{*}\end{array}$} & \multicolumn{2}{|c|}{$\begin{array}{l}\chi^{2}=0.62, P= \\
0.25\end{array}$} \\
\hline $\begin{array}{l}\text { Age } \\
\leq 20\end{array}$ & 42 & $16.7(7)$ & 72 & $13.9(10)$ & 44 & $18.2(8)$ & 158 & $\begin{array}{l}15.8 \\
(25)\end{array}$ \\
\hline $21-30$ & 20 & $20.0(4)$ & 70 & $5.7(4)$ & 22 & $36.4(8)$ & 112 & $\begin{array}{l}14.3 \\
(16)\end{array}$ \\
\hline $31-40$ & 48 & $12.5(6)$ & 28 & $21.4(6)$ & 12 & $16.7(2)$ & 88 & $\begin{array}{l}15.9 \\
(14)\end{array}$ \\
\hline$\otimes 40$ & 80 & $17.5(14)$ & 46 & $26.1(12)$ & 16 & $12.5(2)$ & 142 & $\begin{array}{l}19.7 \\
(28)\end{array}$ \\
\hline $\begin{array}{l}\text { Level of } \\
\text { Significance }\end{array}$ & \multicolumn{2}{|c|}{$\chi^{2}=0.86, P=0.84$} & \multicolumn{2}{|c|}{$\chi^{2}=10.83, P=0.01^{*}$} & \multicolumn{2}{|c|}{$\chi^{2}=3.54, P=0.32$} & \multicolumn{2}{|c|}{$\begin{array}{l}X^{2}=1.40, P= \\
0.70\end{array}$} \\
\hline $\begin{array}{l}\text { Fever status } \\
\text { Febrile }\end{array}$ & 23 & $87.0(20)$ & 34 & $82.4(28)$ & 18 & $88.9(16)$ & 75 & $\begin{array}{l}85.3 \\
(64)\end{array}$ \\
\hline Afebrile & 167 & $6.6(11)$ & 182 & $2.2(4)$ & 76 & $5.3(4)$ & 425 & $4.5(19)$ \\
\hline $\begin{array}{l}\text { Level of } \\
\text { Significance }\end{array}$ & \multicolumn{2}{|c|}{$\chi^{2}=95.03, P \otimes 0.001^{*}$} & \multicolumn{2}{|c|}{$\begin{array}{l}\chi^{2}=145.84, P \otimes \\
0.001^{*}\end{array}$} & \multicolumn{2}{|c|}{$\begin{array}{l}\chi^{2}=60.76, P \otimes \\
0.001^{*}\end{array}$} & \multicolumn{2}{|c|}{$\begin{array}{l}\chi^{2}=300.42, P 区 \\
0.001^{*}\end{array}$} \\
\hline $\begin{array}{l}\text { Clinical } \\
\text { signs } \\
\text { Yes }\end{array}$ & 39 & $20.5(8)$ & 32 & $6.2(2)$ & 30 & $33.3(10)$ & 101 & $\begin{array}{l}19.8 \\
(20)\end{array}$ \\
\hline No & 127 & $15.0(19)$ & 144 & $13.9(20)$ & 44 & $13.6(6)$ & 315 & $\begin{array}{l}14.3 \\
(45)\end{array}$ \\
\hline
\end{tabular}




\begin{tabular}{|c|c|c|c|c|c|c|c|c|}
\hline \multirow[t]{2}{*}{ Categories } & \multicolumn{2}{|c|}{$\begin{array}{l}\text { HIV } \\
\text { Positive }\end{array}$} & \multicolumn{2}{|c|}{$\begin{array}{l}\text { Community HIV } \\
\text { Negative }\end{array}$} & \multicolumn{2}{|c|}{$\begin{array}{l}\text { OPD } \\
\text { HIV Negative }\end{array}$} & \multicolumn{2}{|c|}{ Overall } \\
\hline & $\mathbf{N}$ & $\begin{array}{l}\text { Malaria } \\
\text { parasite } \\
\text { positive \% (n) }\end{array}$ & $\mathbf{N}$ & $\begin{array}{l}\text { Malaria } \\
\text { parasite } \\
\text { positive \% } \\
\text { (n) }\end{array}$ & $\mathbf{N}$ & $\begin{array}{l}\text { Malaria } \\
\text { parasite } \\
\text { positive \% } \\
\text { (n) }\end{array}$ & $\mathbf{N}$ & $\begin{array}{l}\text { Malaria } \\
\text { parasite } \\
\text { positive } \\
\%(n)\end{array}$ \\
\hline $\begin{array}{l}\text { Level of } \\
\text { Significance }\end{array}$ & \multicolumn{2}{|c|}{$\chi^{2}=0.67, P=0.27$} & \multicolumn{2}{|c|}{$X^{2}=1.39, P=0.19$} & \multicolumn{2}{|c|}{$\begin{array}{l}\chi^{2}=4.09, P= \\
0.04^{*}\end{array}$} & \multicolumn{2}{|c|}{$\begin{array}{l}\chi^{2}=1.76, P= \\
0.12\end{array}$} \\
\hline $\begin{array}{l}\text { Anaemic } \\
\text { status } \\
\text { Anaemic }\end{array}$ & 146 & $17.1(25)$ & 86 & $25.6(22)$ & 51 & $35.3(18)$ & 283 & $\begin{array}{l}23.0 \\
(65)\end{array}$ \\
\hline $\begin{array}{l}\text { Non- } \\
\text { anaemic }\end{array}$ & 44 & $13.6(6)$ & 130 & $7.7(10)$ & 43 & $4.7(2)$ & 217 & $8.3(18)$ \\
\hline $\begin{array}{l}\text { Level of } \\
\text { significance }\end{array}$ & \multicolumn{2}{|c|}{$\chi^{2}=0.22, P=0.41$} & \multicolumn{2}{|c|}{$\begin{array}{l}\chi^{2}=10.57, P= \\
0.001^{*}\end{array}$} & \multicolumn{2}{|c|}{$\begin{array}{l}\chi^{2}=12.36, P \otimes \\
0.001^{*}\end{array}$} & \multicolumn{2}{|c|}{$\begin{array}{l}\chi^{2}=16.74, P \otimes \\
0.001^{*}\end{array}$} \\
\hline 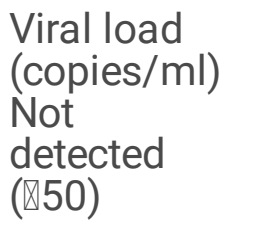 & 141 & $17.0(24)$ & & & & & 141 & $\begin{array}{l}17.0 \\
(24)\end{array}$ \\
\hline$\geq 50$ & 41 & $14.6(6)$ & & & & & 41 & $14.6(6)$ \\
\hline $\begin{array}{l}\text { Level of } \\
\text { Significance }\end{array}$ & \multicolumn{4}{|c|}{$\chi^{2}=0.13, P=0.46$} & & & \multicolumn{2}{|c|}{$\begin{array}{l}\chi^{2}=0.13, P= \\
0.46\end{array}$} \\
\hline $\begin{array}{l}\text { CD4 T-cell } \\
\text { count } \\
\text { (cells/ } \mu \mathrm{l}) \\
\bigotimes 200\end{array}$ & 15 & $6.7(1)$ & & & & & 15 & $6.7(1)$ \\
\hline $200-500$ & 51 & $21.6(11)$ & & & & & 51 & $\begin{array}{l}21.6 \\
(11)\end{array}$ \\
\hline$\varangle 500$ & 40 & $15.0(6)$ & & & & & 40 & $15.0(6)$ \\
\hline $\begin{array}{l}\text { Level of } \\
\text { significance }\end{array}$ & \multicolumn{4}{|c|}{$\chi^{2}=2.00, P=0.36$} & & & \multicolumn{2}{|c|}{$\begin{array}{l}\chi^{2}=2.00, P= \\
0.36\end{array}$} \\
\hline $\begin{array}{l}\text { ART usage } \\
\text { Yes }\end{array}$ & 186 & $16.7(31)$ & & & & & 186 & $\begin{array}{l}16.7 \\
(31)\end{array}$ \\
\hline No & 4 & $0.0(0)$ & & & & & 4 & $0.0(0)$ \\
\hline
\end{tabular}




\begin{tabular}{|c|c|c|c|c|c|c|c|c|}
\hline \multirow[t]{2}{*}{ Categories } & \multicolumn{2}{|c|}{$\begin{array}{l}\text { HIV } \\
\text { Positive }\end{array}$} & \multicolumn{2}{|c|}{$\begin{array}{l}\text { Community HIV } \\
\text { Negative }\end{array}$} & \multicolumn{2}{|c|}{$\begin{array}{l}\text { OPD } \\
\text { HIV Negative }\end{array}$} & \multicolumn{2}{|c|}{ Overall } \\
\hline & $\mathbf{N}$ & $\begin{array}{l}\text { Malaria } \\
\text { parasite } \\
\text { positive \% (n) }\end{array}$ & $\mathbf{N}$ & $\begin{array}{l}\text { Malaria } \\
\text { parasite } \\
\text { positive \% } \\
\text { (n) }\end{array}$ & $\mathbf{N}$ & $\begin{array}{l}\text { Malaria } \\
\text { parasite } \\
\text { positive \% } \\
\text { (n) }\end{array}$ & $\mathbf{N}$ & $\begin{array}{l}\text { Malaria } \\
\text { parasite } \\
\text { positive } \\
\%(n)\end{array}$ \\
\hline $\begin{array}{l}\text { Level of } \\
\text { significance }\end{array}$ & \multicolumn{4}{|c|}{$\chi^{2}=0.79, P=0.48$} & & & \multicolumn{2}{|c|}{$\begin{array}{l}\chi^{2}=0.79, P= \\
0.48\end{array}$} \\
\hline $\begin{array}{l}\text { ART } \\
\text { duration } \\
\otimes 1\end{array}$ & 36 & $13.9(5)$ & & & & & 36 & $13.9(5)$ \\
\hline $1-3$ & 61 & $18.0(11)$ & & & & & 61 & $\begin{array}{l}18.0 \\
(11)\end{array}$ \\
\hline$>3$ & 89 & $16.9(15)$ & & & & & 89 & $\begin{array}{l}16.9 \\
(15)\end{array}$ \\
\hline $\begin{array}{l}\text { Level of } \\
\text { significance }\end{array}$ & \multicolumn{4}{|c|}{$X^{2}=0.28, P=0.86$} & & & \multicolumn{2}{|c|}{$\begin{array}{l}\chi^{2}=0.28, P= \\
0.86\end{array}$} \\
\hline $\begin{array}{l}\text { ART } \\
\text { regimen } \\
\text { First line }\end{array}$ & 171 & $17.5(30)$ & & & & & 171 & $\begin{array}{l}17.5 \\
(30)\end{array}$ \\
\hline Second line & 15 & $6.7(1)$ & & & & & 15 & $6.7(1)$ \\
\hline $\begin{array}{l}\text { Level of } \\
\text { significance }\end{array}$ & \multicolumn{4}{|c|}{$X^{2}=1.21, P=0.24$} & & & \multicolumn{2}{|c|}{$\begin{array}{l}\chi^{2}=1.21, P= \\
0.24\end{array}$} \\
\hline
\end{tabular}

\section{Intestinal Parasite Prevalence}

Among the 500 participants, 65 (13.0\%) were positive for IPIs with a higher occurrence of protozoa (10.4\%) than helminths (3.8\%). The prevalence of IPs was highest among PLWH (23.7\%) and least among HIV negative controls in the communities (5.6\%) and the difference was significant at $\chi^{2}=31.73$, $\mathrm{P} \otimes 0.001$ as shown in Fig. 3.

Detected protozoa were Cryptosporidium species and Entamoeba histolytica while detected helminths were Ascaris lumbricoides, Schistosoma mansoni, hookworm, Dipylidium caninum, Hymenolepis nana and Taenia species. When comparing infection prevalence among the different groups, PLWH were the most infected both with helminths (6.8\%) and protozoa (19.5\%) and the differences were statistically significant $\left(\chi^{2}=9.74, P=0.008 ; \chi^{2}=28.78, P \otimes 0.001\right.$ respectively $)$ as shown in Table 4. 
Table 4

Prevalence of intestinal parasites in the study population

\begin{tabular}{|c|c|c|c|c|c|}
\hline $\begin{array}{l}\text { Parasites } \\
\text { Species }\end{array}$ & $\begin{array}{l}\text { HIV } \\
\text { Positive } \\
\text { n (\%) }\end{array}$ & $\begin{array}{l}\text { Community HIV } \\
\text { Negative } \\
\mathrm{n}(\%)\end{array}$ & $\begin{array}{l}\text { OPD } \\
\text { HIV } \\
\text { Negative } \\
\mathrm{n}(\%)\end{array}$ & $\begin{array}{l}\text { Overall } \\
\text { population } \\
\mathrm{n}(\%)\end{array}$ & $\begin{array}{l}\text { Chi } \\
\text { Square } \\
\text { P Value }\end{array}$ \\
\hline \multicolumn{6}{|l|}{ Protozoa } \\
\hline $\begin{array}{l}\text { At least one } \\
\text { protozoa }\end{array}$ & 37 (19.5) & $10(4.6)$ & $4(4.3)$ & $51(10.2)$ & $\begin{array}{l}\chi^{2}= \\
28.78 \\
P \otimes \\
0.001^{*}\end{array}$ \\
\hline $\begin{array}{l}\text { Cryptosporidium } \\
\text { spp }\end{array}$ & $32(16.8)$ & $10(4.6)$ & $4(4.3)$ & $46(9.2)$ & $\begin{array}{l}\chi^{2}= \\
21.63 \\
P \otimes \\
0.001^{*}\end{array}$ \\
\hline $\begin{array}{l}\text { Entamoeba } \\
\text { histolytica }\end{array}$ & $6(3.2)$ & $0(0.0)$ & $0(0.0)$ & $6(1.2)$ & $\begin{array}{l}X^{2}=9.96 \\
P=0.01^{*}\end{array}$ \\
\hline \multicolumn{6}{|l|}{ Helminths } \\
\hline $\begin{array}{l}\text { At least one } \\
\text { helminth }\end{array}$ & $13(6.8)$ & $2(0.9)$ & $4(4.3)$ & $19(3.8)$ & $\begin{array}{l}X^{2}=9.74 \\
P= \\
0.008^{*}\end{array}$ \\
\hline $\begin{array}{l}\text { Ascaris } \\
\text { lumbricoides }\end{array}$ & $4(2.1)$ & $0(0.0)$ & $2(2.1)$ & $6(1.2)$ & $\begin{array}{l}\chi^{2}=4.63 \\
P=0.09\end{array}$ \\
\hline Hookworm & $5(2.6)$ & $0(0.0)$ & $0(0.0)$ & $5(1.0)$ & $\begin{array}{l}X^{2}=8.28 \\
P=0.02^{*}\end{array}$ \\
\hline $\begin{array}{l}\text { Schistosoma } \\
\text { mansoni }\end{array}$ & $1(0.5)$ & $0(0.0)$ & $2(2.1)$ & $3(0.6)$ & $\begin{array}{l}\chi^{2}=4.98 \\
P=0.08\end{array}$ \\
\hline $\begin{array}{l}\text { Strongyloides } \\
\text { stercoralis }\end{array}$ & $1(0.5)$ & $0(0.0)$ & $0(0.0)$ & $1(0.2)$ & $\begin{array}{l}\chi^{2}=1.64 \\
P=0.44\end{array}$ \\
\hline Tapeworm & $2(1.1)$ & $2(0.9)$ & $0(0.0)$ & $4(0.8)$ & $\begin{array}{l}\chi^{2}=0.96 \\
P=0.62\end{array}$ \\
\hline
\end{tabular}

Infection with Cryptosporidium species was the most common IPs identified in the study population $(9.2 \%, 46)$, followed by Ascaris lumbricoides $(1.2 \%, 6)$ and Entamoeba histolytica $(1.2 \%, 6)$ and then hookworm $(1.0 \%, 5)$. When compared with their counterparts, PLWH had the highest prevalence of 
Cryptosporidium species (16.8\%), Entamoeba histolytica (3.2\%) and hookworm (2.6\%) and the differences were significant $(P \otimes 0.001, P=0.01, P=0.02$ respectively) as shown in Table 4.

\section{Malaria /intestinal Parasite Co-infections}

Malaria/IPs co-infection prevalence was 2.2\% (11) and was significantly higher among PLWH (3.7\%) when compared with HIV negative participants in the communities $\left(X^{2}=5.96, P=0.04\right)$ (Fig. 4). MP/IPs co-infection was not observed among HIV negative patients at the OPD of the hospital. Among PLWH, malaria/IPs co-infection was comparable among males (4.0\%) and females (3.6\%). On the other hand, the prevalence was significantly higher $\left(X^{2}=8.08, P=0.04\right)$ in those $21-30$ years old $(15.0 \%)$ than their counterparts as revealed in Table 5. Those who presented with fever had significantly higher prevalence $(P \otimes 0.001)$ of malaria/IPs co-infection (21.7\%) than their afebrile counterparts (1.2\%). While patients who attained viral load suppression had none significant $(P=0.50)$ lower prevalence $(3.8 \%)$, those who have been on ART for more than three years showed a significantly lower $(P=0.03)$ prevalence of malaria/IPs co-infection (1.1\%) than their respective counterpart as shown in Table 5. 
Table 5

Malaria/IP co-infection prevalence among PLWH with respect to sociodemographic and clinical data

\begin{tabular}{|c|c|c|c|}
\hline \multirow[t]{2}{*}{ Categories } & \multicolumn{2}{|c|}{ PLWH } & \multirow{2}{*}{$\begin{array}{l}\text { Level of Significance } \\
\left(\chi^{2}, P\right)\end{array}$} \\
\hline & $\mathrm{N}$ & $\begin{array}{l}\text { Malaria/IP positive } \\
\%(n)\end{array}$ & \\
\hline $\begin{array}{l}\text { Sex } \\
\text { Male }\end{array}$ & 50 & $4.0(2)$ & \multirow[t]{2}{*}{$\begin{array}{l}\chi^{2}=0.02 \\
P=0.59\end{array}$} \\
\hline Female & 140 & $3.6(5)$ & \\
\hline $\begin{array}{l}\text { Age } \\
\leq 20\end{array}$ & 42 & $2.4(1)$ & \multirow[t]{4}{*}{$\begin{array}{l}\chi^{2}=8.08 \\
P=0.04\end{array}$} \\
\hline $21-30$ & 20 & $15.0(3)$ & \\
\hline $31-40$ & 48 & $2.1(1)$ & \\
\hline$\nabla 40$ & 80 & $2.5(2)$ & \\
\hline $\begin{array}{l}\text { Fever status } \\
\text { Febrile }\end{array}$ & 23 & $21.7(5)$ & \multirow[t]{2}{*}{$\begin{array}{l}\chi^{2}=24.04 \\
P \square 0.001\end{array}$} \\
\hline Afebrile & 167 & $1.2(2)$ & \\
\hline $\begin{array}{l}\text { Clinical signs } \\
\text { Yes }\end{array}$ & 39 & $2.6(1)$ & \multirow[t]{2}{*}{$\begin{array}{l}\chi^{2}=0.15 \\
P=0.57\end{array}$} \\
\hline No & 128 & $3.9(5)$ & \\
\hline $\begin{array}{l}\text { Anaemic status } \\
\text { Anaemic }\end{array}$ & 146 & $4.1(6)$ & \multirow[t]{2}{*}{$\begin{array}{l}\chi^{2}=0.32 \\
P=0.48\end{array}$} \\
\hline Non-anaemic & 44 & $2.3(1)$ & \\
\hline 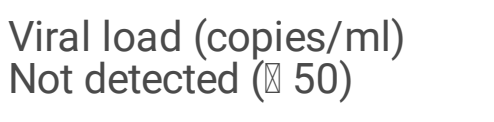 & 141 & $4.3(6)$ & \multirow[t]{2}{*}{$\begin{array}{l}\chi^{2}=0.28 \\
P=0.50\end{array}$} \\
\hline$\geq 50$ & 41 & $2.4(1)$ & \\
\hline $\begin{array}{l}\text { CD4 T-cell count (cells/ } / \text { l) } \\
\otimes 200\end{array}$ & 15 & $6.7(1)$ & \multirow[t]{3}{*}{$\begin{array}{l}\chi^{2}=3.22 \\
P=0.20\end{array}$} \\
\hline $200-500$ & 51 & $7.8(4)$ & \\
\hline$\otimes 500$ & 40 & $0.0(0)$ & \\
\hline $\begin{array}{l}\text { ART usage } \\
\text { Yes }\end{array}$ & 186 & $3.8(7)$ & \multirow[t]{2}{*}{$\begin{array}{l}\chi^{2}=0.64 \\
P=0.86\end{array}$} \\
\hline No & 4 & $0.0(0)$ & \\
\hline $\begin{array}{l}\text { ART duration (years) } \\
\otimes 1\end{array}$ & 36 & $11.1(4)$ & $\begin{array}{l}\chi^{2}=7.12 \\
P=0.03\end{array}$ \\
\hline
\end{tabular}




\begin{tabular}{|c|c|c|c|}
\hline $1-3$ & 61 & $3.3(2)$ & \\
\hline$\otimes 3$ & 89 & $1.1(1)$ & \\
\hline $\begin{array}{l}\text { ART regimen } \\
\text { First line }\end{array}$ & 171 & $3.5(6)$ & $\begin{array}{l}\chi^{2}=0.38 \\
P=0.45\end{array}$ \\
\hline Second line & 15 & $6.7(1)$ & \\
\hline
\end{tabular}

\section{Haematological Abnormalities And Risk Factors Of Anaemia}

Anaemia and leucopenia were found to be significantly higher ( $P \otimes 0.001, P \otimes 0.001$ respectively) among PLWH (76.8\% and 55.1\% respectively) when compared with their HIV negative equivalent. The prevalence of thrombocytopenia was comparable among the different groups. PLWH had the lowest prevalence of microcytosis $(43 \%, 80)$ and the difference was statistically significant $\left(X^{2}=45.94, P \otimes 0.001\right)$ as shown in Fig. 5.

As shown in Fig. 6, there was a significant difference ( $P \otimes 0.001)$ when comparing haemoglobin values between the different infection categories; as those triple infected had the lowest haemoglobin value when compared with dual and single infected patients. A multinomial regression analysis was used to determine the factors associated with anaemia. As shown in Table 6, being $21-30$ years old $(P=0.029)$, a male $(P<0.001)$, malaria parasite positive $(P<0.001)$, HIV positive $(P<0.001)$ and being febrile $(P<$ 0.001 ) were significant risk factors of anaemia in the study population. 
Table 6

Risk factors associated with anaemia in the study population

\begin{tabular}{|c|c|c|c|}
\hline Parameters & Odds ratio (OR) & $95 \% \mathrm{Cl}$ & P-value \\
\hline \multicolumn{4}{|l|}{${ }^{\mathrm{a}}$ Age group (years) } \\
\hline$\leq 20$ & 0.792 & $0.499-1.257$ & 0.32 \\
\hline $21-30$ & 0.571 & $0.346-0.944$ & $0.03^{*}$ \\
\hline $31-40$ & 0.886 & $0.515-1.526$ & 0.66 \\
\hline${ }^{b}$ Male & 2.601 & $1.779-3.802$ & $\otimes 0.001^{*}$ \\
\hline${ }^{\mathrm{c}}$ Febrile & 0.330 & $0.186-0.585$ & $\nabla 0.001^{*}$ \\
\hline dHIV positive & 0.239 & $0.159-0.358$ & $\nabla 0.001^{*}$ \\
\hline e MP positive & 0.292 & $0.167-0.508$ & $\nabla 0.001^{*}$ \\
\hline fMP/IPs co-infection & 0.285 & $0.126-1.838$ & 0.28 \\
\hline gHelminth positive & 0.591 & $0.221-1.580$ & 0.29 \\
\hline${ }^{h}$ Protozoan positive & 0.825 & $0.456-1.492$ & 0.52 \\
\hline 'Leucopenia & 0.573 & $0.387-0.848$ & $0.01^{*}$ \\
\hline \multicolumn{4}{|c|}{ *Statistically significant P-value } \\
\hline \multicolumn{4}{|c|}{ aAge group: $\leq 20$ years $=1 ; 21-30$ years $=2 ; 30-40$ years $=3 ; \geq 41$ years $=4$} \\
\hline \multicolumn{4}{|l|}{${ }^{\mathrm{b}}$ Male $=1 ;$ Female $=2$} \\
\hline \multicolumn{4}{|c|}{${ }^{\mathrm{c}}$ Febrile $=1 ;$ Afebrile $=2$} \\
\hline \multicolumn{4}{|c|}{ dHIV positive $=1 ;$ HIV negative $=2$} \\
\hline \multicolumn{4}{|c|}{${ }^{\mathrm{e}} \mathrm{MP}$ positive $=1 ; \mathrm{MP}$ negative $=2$} \\
\hline \multicolumn{4}{|c|}{${ }^{f} \mathrm{MP} / \mathrm{IPs}$ co-infection $=1 ;$ No MP/IPs co-infection $=2$} \\
\hline \multicolumn{4}{|c|}{ gHelminth positive $=1$; Helminth negative $=2$} \\
\hline \multicolumn{4}{|c|}{${ }^{h}$ Protozoan positive $=1 ;$ Protozoan negative $=2$} \\
\hline 'Leucopenia $=1$; No l $\epsilon$ & penia $=2$ & & \\
\hline
\end{tabular}




\section{Discussion}

Parasitic infections (especially malaria and intestinal parasites) are major public health concern in PLWH in developing countries, particularly Sub-Saharan Africa which has been reported to have the highest burden of HIV [2]. This study was a hospital- and community-based cross-sectional study carried out to determine the prevalence of malaria and intestinal parasites among PLWH and HIV negative individuals in Buea, in South West Region of Cameroon.

In the present study, the overall prevalence of malaria parasite was $16.6 \%$, irrespective of the HIV status of the participants. This result is lower than the recently reported national prevalence (24.4\%) [30]. This decrease could be attributed to the restless efforts of the Cameroon government over the years to implement malaria control strategies so as to curb down malaria morbidity and mortality on the entire territory; knowing that Cameroon comes fourth on the list of countries that account for the burden of malaria in the WHO African Region [5].

The prevalence of malaria parasite among PLWH was $16.4 \%$, which is high compared to the prevalence reported in PLWH in other areas of the South West Region of Cameroon. This included the $7.8 \%$ reported in Limbe [10] and 2.3\% in Buea [31] It was also observed in this study that despite the efforts made to scale up insecticide-treated nets (ITNs) distribution so that universal coverage can be attained, coverage remains low and this could have contributed to this rise in malaria prevalence among PLWH.

The prevalence of malaria parasite was higher in HIV negative participants (21.3\%) than in their HIV positive counterparts. This finding is in line with studies carried out in Cameroon [10] and Ethiopia [32] which also reported a higher malaria parasite prevalence in HIV negative participants at the OPD of the hospital when compared with their HIV positive counterparts. Although HIV positive patients are considered as a high-risk group for malaria, malaria is not the most frequent diagnosis in these patients. Majority of the HIV positive patients were under a combination therapy of ART and cotrimoxazole which probably protected them against infections. Cotrimoxazole has been reported in several studies to have some protective effect against parasitic infections, both in clinical trials $[33,34,35]$ and in the hospital [22].

In this study, while MP prevalence was higher among HIV patients who did not attain viral suppression (viral load $\geq 50$ copies/ml of blood), the majority of the HIV positive patients attained viral suppression due to their adherence to ART over the years. This is in line with recent findings in western Kenya [36] and further confirms the benefits of adherence to ART.

It is worth noting that HIV negative participants from the Buea communities had the least malaria parasite prevalence $(14.8 \%)$ when compared with their counterparts. These malaria asymptomatic carriers are those who greatly fuel the transmission of malaria parasite in the community. This low prevalence shows that strategies implemented by the Cameroon government over the years are effective as this prevalence is lower than the reported national prevalence of malaria in 2018 [5]. 
Fever is one of the most common clinical sign and characteristic feature of both parasitic and microbial infections. In this study, febrile status of most of the participants irrespective of their study group was attributed to the presence of malaria parasite. This is in line with other studies carried out in Cameroon [10] and in Gabon [37] Also feverish PLWH had the highest prevalence of MP/IPs co-infection asserting the fact that fever remains an important feature in the clinical diagnosis of infections.

The prevalence of IPs in Cameroon varied from 27.8\% in 2012 [38], to 14.6\% in 2013 [39] and $13.0 \%$ in this present study. Considering the HIV status, PLWH had the highest prevalence of IPs $(23.7 \%)$ when compared with their HIV negative counterparts. This prevalence among PLWH is lower than the $59.52 \%$ [39] and 57.48\% [40] reported among HIV patients in the Centre and West Regions of Cameroon respectively. This observed decrease in the prevalence of IPs among HIV patients may be accredited to attainment of viral suppression by a majority as well as their better awareness of IPs and their causes. It may also be due to the improvement in care provided to PLWH by the government of Cameroon by ensuring a constant supply and provision of ART free of charge.

In line with other studies in Cameroon [15], Ethiopia [41] and in China [42], the prevalence of protozoa was higher than that of helminths. Both the HIV positive and HIV negatives were infected with Cryptosporidium spp; but PLWH had the highest prevalence of Cryptosporidium spp (16.8\%). The prevalence of Cryptosporidium spp in this study is lower unlike the 44\% reported earlier in Cameroon [15] and the 79.0\%, in South Africa [43]. Cryptosporidium spp is recognized as an opportunistic parasite in HIV/AIDS patients because it tends to be present when the CD $4+T$ cell counts are below 200 cells $/ \mu \mathrm{L}$. Hence, this decreased prevalence among HIV patients could be attributed to the success of intervention efforts over the years in reducing the significance of opportunistic intestinal parasites in this at risk group. Hookworm was the most prevalent helminth among HIV patients with a prevalence of $2.6 \%$. A prevalence of $2.53 \%$ and $2.7 \%$ have also been reported in studies carried out in Yaounde, [40] and in Buea [44] Cameroon.

This study revealed 3.7\% of PLWH were co-infected with MP and IPs. Limited information is available on co-infection among PLWH in Cameroon; but when compared with other studies carried out among children $[18,45,46]$, the prevalence is low. The mean age (35.5 \pm 16.5 years) of PLWH as well as the undetectable viral load observed in the majority of these patients could account for this low prevalence. The lower the viral load, the faster the patient's immune system will recover, increasing the chances of fighting any infection easily. In addition, majority of this patients have been on ART regularly for more than a year which probably could have contributed in boosting their immune system against these parasites.

The overall prevalence of anaemia of $56.6 \%$ irrespective of their HIV status is higher than the $52.1 \%$ reported in previous study in the Mount Cameroon area [10].This increase may be attributed to the rise in malaria parasite prevalence observed in the study when compared with previous studies in the area. Several authors have reported malaria parasite as a risk factor of anaemia $[47,48]$ due to the 
pathogenesis of the infection that involves the destruction of both infected and non-infected red blood cells.

PLWH had a higher prevalence of anaemia (76.8\%) when compared with their HIV negative counterparts. This prevalence is higher than the less than $56.0 \%$ reported in several studies carried out among PLWH $[49,9,50,51,52,53,10]$. The reason for the observed difference might be due to the heterogeinity of the study population as these previously reported anaemia prevalences were those of children or adults HIV patients, but this study included both children and adult participants. Malaria and HIV positivity, as well as fever were identified in this study as risk factors of anaemia and therefore contributed significantly to the high prevalence of anaemia. Mixed nutritional deficiencies (iron, folic acid, or vitamin B12), though not investigated in this study, could have contributed to this high prevalence of anaemia as reported by a study carried out by Volberding et al., [54]. A direct comparison of prevalence of anaemia in different studies is difficult as the study population, inclusion and exclusion criteria and anaemia definitions were different.

Parasites like the malaria parasites and intestinal parasites have long been recognized as major contributors to reduced haemoglobin levels in endemic countries like Cameroon, thereby causing anaemia. In addition, PLWH on ART (containing Zidovudine) have been reported to influence the haemoglobin level in previous studies $[55,56,57,9,10]$. In this study, patients infected with both HIV, malaria and IPs had a significantly lower mean haemoglobin value $(10.6 \pm 1.21 \mathrm{~g} / \mathrm{dL})$. This in line with previous studies which reported though among children, low haemoglobin values in patients with coinfections when compared with those with single infections $[58 ; 59]$.

The higher prevalence of Leucopenia observed among PLWH (55.5\%) when compared with HIV negative counterparts has been reported in studies carried out in Ethiopia [60], Nigeria [61] as well as in Cameroon [50]. This high prevalence could be associated with profound immunodeficiency as more than half of PLWH had CD 4 T cell count $<500$ cells/ $\mu \mathrm{L}$ of blood. While observation from this study revealed no significant difference in the prevalence of thrombocytopenia between PLWH and HIV negative participants, a decrease has been reported in other studies after ART introduction [62,63]. Microcytosis was present among PLWH but the prevalence was lower (43\%) when compared with their HIV negative counterparts. As PLWH attend an HIV specialty unit, they often receive medical talks with respect to their immunity, nutritional status and general wellbeing as part of the case management. This could have contributed to the low prevalence of microcytosis in this group which is often due to iron deficiency.

Even though different types of HIV and MP species have been reported in Cameroon, limited knowledge exist on the occurrence of MP/IPS co-infection among PLWH. Findings of this study fills some of the gaps although it has its limitations. Notwithstanding, the fact that ART-naïve HIV infected patients were not included in the study, nutritional deficiency as a cause of anaemia was not investigate, as well as the cross sectional nature of the study that provides a picture at a point in time, the findings are valuable to policy makers in guiding the management of these conditions. Future longitudinal studies on the impact of iron supplementation and vitamin B12 levels among PLWH may be of great relevance. 


\section{Conclusions}

From this study, it can be concluded that malaria parasite and intestinal parasites (especially protozoa) remain a public health concern among PLWH with higher occurrences of both MP, IPs and MP/IP coinfections than in their HIV negative counterparts in the communities. Though a good majority of the HIV patients in the study had attained viral suppression, they are still more vulnerable to some of the parasites than HIV negative individuals and therefore can constitute a good source of contamination in their various communities. To achieve HIV epidemic control in 2021, the Cameroon government needs to intensify the implementation of the various strategies. Anaemia was the most prevalent haematological abnormality among HIV patients and gets exacerbated even with the viral load suppression in the population of PLWH. Hence, there is need for constant monitoring of the health status as well as nutritional status of this at risk group to better manage them.

\section{Abbreviations}

AIDS

Acquired immune deficiency syndrome;

ANOVA

Analysis of variance

ART

Anti-retroviral Therapy;

CAMPHIA

Cameroon population-based HIV impact assessment;

CD4 T

T-lymphocyte cell bearing CD4 receptors;

$\mathrm{Cl}$

Confidence interval

EDTA

Ethylene diaminetetraacetic acid

IPs

Intestinal parasites

IPI

Intestinal parasite infection

$\mathrm{Hb}$

Haemoglobin

HIV

Human Immunodeficiency Virus

$\mathrm{MCH}$

Mean corpuscular haemoglobin

$\mathrm{MCHC}$ 
Mean corpuscular haemoglobin concentration

MCV

Mean corpuscular volume

MP

Malaria parasite

MPV

Mean platelet volume

OPD

Out Patient Department

$P$

Probability level

PLT

Platelets

PLWH

People Living With HIV

RBCs

Red Blood Cells

RDW

Red Cell Distribution Width

RHB

Regional Hospital Buea

SD

Standard Deviation

SPSS

Software Package of Social Sciences

UNAIDS

Joint United Nations Programme on HIV/AIDS

$\mathrm{VL}$

Viral Load

WBC

White Blood Cell

WHO

World Health Organization

\section{Declarations}

\section{Ethics approval and consent to participants}

An administrative clearance was obtained from the South West Regional Delegation of Public Health, Buea, followed by an ethical clearance from the Institutional Review Board hosted by the Faculty of Health Sciences of the University of Buea (Reference Number: 661-09). Pre- and post-HIV counselling was 
done by competent personnel. All test procedures were done free of charge with confidentiality maintained. Participation in the study was voluntary. Participants could ask questions and their decisions not to participate in the study were fully respected and this did not affect the services provided to them in any way. They were also free to withdraw from the study at any time that they wished. All information collected from the field remained strictly confidential.

\section{Consent for publication}

Not applicable

\section{Availability of data and materials}

All datasets on which the conclusions of the research rely are presented in this paper. However, data is available from the corresponding author on reasonable request.

\section{Competing interests}

The authors declare that they have no competing interests.

\section{Funding}

This work was supported by the special fund for research and modernization given to authors by the Government of Cameroon. With the availability of the funds, the authors conveniently designed the work. The funds supported transportation to the field for sample collection, purchase of supplies and equipment for sample analyses.

\section{Authors' Contributions}

SMS conceived the study, designed and coordinated the field work, data collection and analysis as well as the write-up of the manuscript. IUNS participated in data analysis, supervision and revision of the manuscript. MMT was involved in data collection, interview of the participants and data analysis. HKK designed the study and revised the manuscript critically for important intellectual content. All authors read and approved the manuscript.

\section{Acknowledgments}

We thank all the participants (both children and adults) who took part in the study as well as to the staff of the Regional Hospital Buea, especially the staff at the Unit in-charge of patients with HIV (UPEC) and the laboratory of the Regional Hospital Buea for letting the practical aspect of this study go on smoothly with no incidents, for their guidance and critical comments concerning the care given to their patients. We are also thankful to the Buea community inhabitants who consented and parents who consented for their children to participate in the study.

\section{References}


1. Bangert M. The cross-cutting contribution of the end of neglected tropical diseases to the sustainable development goals. Infectious Diseases of Poverty. 2017;6(1):73.

2. UNAIDS. UNAIDS report on the global AIDS epidemic 2018, Geneva, Switzerland.

3. Country Operational Plan (COP), Cameroon. 2019. Strategic Direction Summary. Accessed 07 Apr 2020.

4. World Health Organization. World Malaria Report 2019. Cameroon, African Region 2019. Accessed 28 Feb 2020 .

5. World Health Organization. World Malaria Report 2018. Cameroon, African Region 2018. Accessed 04 Mar 2020.

6. Nkuo-Akenji T. Tevoufouet EE, Nzang F. Fon E, Ebong IN. HIV/AIDS and malaria in pregnant women from Cameroon short running title: HIV, malaria in pregnancy. Afr J Health Sci. 2011;18:105-9.

7. Njunda LA. Kamga HLF, Nsagha DS. Assoh JCN, Kwenti TE. Low malaria prevalence in HIV-positive patients in Bamenda, Cameroon. J Microbiol Res. 2012;2:56-9.

8. Kimbi HK. Njoh DT, Ndamukong KJN,Lehman LG. Malaria in HIV/AIDS patients at different CD4 T cell levels in Limbe, Cameroon. J Bacteriol Parasitol. 2013;4 (164) doi: .

9. Bate B, Kimbi HK, Lum E, Lehman LG, Onyoh EF, Ndip LM, Njabi CM, Tonga C, Wempnje GB, Ndip RN, Bessong PO. Malaria infection and anaemia in HIV-infected children in Mutengene, Southwest Cameroon: a cross sectional study. BMC Infect Dis. 2016;16:523. .

10. Sandie SM, Sumbele IUN, Tasah MM and Kimbi HK. Malaria parasite prevalence and Haematological parameters in HIV seropositive patients attending the regional hospital Limbe, Cameroon: a hospitalbased cross-sectional study. BMC Infect Dis. 2019;19, 988 .

11. Malaria Operational Plan (MOP) FY. 2018 and FY 2019. President's Malaria Initiative Cameroon, 2019. Accessed: 07 Apr 2020.

12. Alemu G. Alelign D, Abossie A. Prevalence of Opportunistic Intestinal Parasites and Associated Factors among HIV Patients while Receiving ART at Arba Minch Hospital in South Ethiopia: A Crosssectional Study. Ethiop J Health Sci 2018; 28(2):147. doi..

13. Webb EL. Ekii AO, Pala P. Epidemiology and immunology of helminth = HIV interactions. Curr Opin HIV AIDS. 2012;7:245-53.

14. Adeleke OA. Yogeswaran P, Wright G. Intestinal helminth infections amongst HIV-infected adults in Mthatha General Hospital, South Africa. Afr J Prm Health Care Fam Med. 2015; 7(1). .

15. Nsagha DS. Njunda AL, Assob NJC. Ayima CW, Tanue EA. kibu OD and Kwenti TE. Intestinal parasitic infections in relation to CD4 + Tcell counts and diarrhea in HIV/AIDS patients with or without antiretroviral therapy in Cameroon. BMC Infect Dis. 2016;16:9. DOI.

16. Nacher M. Singhasivanon P, Yimsamran S. Manibunyong W, Thanyavanich N. Wuthisen R. Intestinal helminth infections are associated with increased incidence of Plasmodium falciparum malaria in Thailand. J Parasitol. 2002;88(1):55-8. 
17. Sokhna C. Le Hesran JY, Mbaye PA. Akiana J, Camara P. Diop M. Increased malaria attacks among children presenting concomitant infections by S. mansoni in Senegal. Malar J. 2004;3:43.

18. Njunda AL. Fon SG, Assob JCN. Nsagha DS, Kwenti TDB, Kwenti TE. Coinfection with malaria and intestinal parasites, and its association with anaemia in children in Cameroon. Infectious Disease of Poverty. 2015;4:43 DOI.

19. Wagnew F, Eshetie S, Alebel A, Tesema C. Kibret GD, Gebrie A. Dessie G, Abajobir AA. Burden of anemia and its association with HAART in HIV infected children in Ethiopia: a systematic review and meta-analysis. BMC Infect Dis. 2019;19 .

20. Brooker S. Akhwale W, Pullan R. Estambale B, Clarke SE. Snow RW. Epidemiology of Plasmodiumhelminth co-infection in Africa: populations at risk, potential impact on anemia and prospects for combining control. Am J Trop Med Hyg.2007;77(6S):88-98.

21. Sumbele IU, Nkemnji GB, Kimbi HK. Soil-transmitted helminths and plasmodium falciparum malaria among individuals living in different agroecosystems in two rural communities in the mount Cameroon area: a cross-sectional study. Infectious diseases of poverty, 2017;6(1), 67. doi..

22. Saracino A. Nacarapa EA, Massinga EAC. Martinelli D, Oliveira C. Antonich A, Galloni D. Ferro JJ, Macome CA, Scacchetti M. Prevalence and clinical features of HIV and malaria co-infection in hospitalized adults in Beira, Mozambique. Malaria Journal 2012, 11:241 .

23. CAMPHIA (Cameroon Population-based HIV Impact Assessment). 2017. Summary sheet: Preliminary findings. CAMPHIA_Cameroon-SS_A4 v13_requests_7.25.18.pdf 2017. Accessed 09 Apr 2020.

24. World Health Organization World Health Organization. Malaria Factsheets 2020. 2020. Accessed 02 Apr 2020.

25. Bryan FJ. The Design and Analysis of Research Studies, University of Otago, Dunedin, New Zealand. Cambridge: Cambridge University Press; 1992.

26. Cheesbrough M. District Laboratory Practice in Tropical Countries, 3Rd ed., part 1. United Kingdom: Cambridge University Press; 2014. p. 178-215.

27. UNAIDS

UNAIDS. Undetectable $=$ Untransmittable. Publich health and HIV load suppression. 2018. Accessed 09 Apr 2020.

28. WHO Nutritional anaemias: tools for effective prevention and control WHO Nutritional anaemias: tools for effective prevention and control. Geneva, Switzerland: World Health Organization Publication; 2017. . Accessed 07 April 2020.

29. Pagana KD, Pagana TJ, Pagana TN. Mosby's Diagnosis and Laboratory Test Reference. 14th ed. St Louis: Elsevier; 2019. Pp 1088.

30. Minsante. Xlième Journée mondiale de lutte contre le paludisme "prêt à vaincre le paludisme" Nous sommes la génération qui peut éliminer le paludisme. Dossier de Presse. Minsante. 2018:1-20.

31. Mbah-Mbole FG, Tufon KA, Meriki DH, Enow-Orock G, Mbah-Mbole P, Njunda LA, Wam Iwoi MD, Kwenti TE. Malaria and human immunodeficiency virus coinfection in febrile patients attending the Regional Hospital of Buea, Southwest region, Cameroon. Int J Adv Med Health Res. 2018;6:46-51. 
32. Alemayehu G, Melaku Z, Abreha T, Alemayehu B, Girma S, Tadesse Y, Gadisa T, Lulseged S, Balcha TT, Hoos D. Teka1 H and Reithinge R.Burden of malaria among adult patients attending general medical outpatient department and HIV care and treatment clinics in Oromia, Ethiopia: a comparative cross-sectional study. Malar J. 2015;14:501.

33. Gasasira AF, Kamya MR, Ochong EO, Vora N, Achan J, Charlebois E, Ruel T, Kateera F, Meya DN, Havlir D, Rosenthal PJ, Dorsey G. Effect of trimethoprim-sulphamethoxazole on the risk of malaria in HIV-infected Ugandan children living in an area of wide spread antifolate resistance. Malar J. 2010;9:177.

34. Walker AS, Forda D, Gilksb CF, Munderic P, Ssalid F, Reide A, Katabiraf E, Grosskurthc H, Mugyenyid P, Hakime J, Darbyshirea JH, Gibba DM. Babikera AG.Daily co-trimoxazole prophylaxis in severely immuno suppressed HIV-infected adults in Africa started on combination antiretroviral therapy: an observational analysis of the DART cohort. Lancet. 2010;37:1278-86.

35. Kapito-Tembo A, Meshnick SR, van Hensbroek MB, Phiri K, Fitzgerald M, Mwapasa V. Marked reduction in prevalence of malaria parasitemia and anemia in HIV-infected pregnant women taking cotrimoxazole with or without sulfadoxine-pyrimethamine intermittent preventive therapy during pregnancy in malawi. J Infect Dis. 2011;203:464-72.

36. Kirinyet JK. An Assessment of Malaria Parasite Density among HIV/AIDS-Subjects at Different Levels of CD4 T-Cells Prior to Antimalarial Therapy at Chulaimbo Sub-County Hospital, Western Kenya. Hindawi. 2019. Article ID 56973837 pages .

37. Bouyou-Akotet M, Offouga C, Mawili-Mboumba D, Essola L, Madoungou B, Kombila M. Falciparum Malaria as an Emerging Cause of Fever in Adults Living in Gabon, Central Africa. BioMed Res Int. 2014. . Article ID 351281.

38. Lehman LG, Kangam L, Nguepi E, Mbenoun ML, Bilong Bilong CF. Study of intestinal parasitic infections associated with HIV infection in Douala, Cameroon. Retrovirology. 2012;9(Suppl 1):P48.

39. Nkenfou CN, Nana CT, Payne VK. Intestinal Parasitic Infections in HIV Infected and Non-Infected Patients in a Low HIV Prevalence Region, West- Cameroon. PLoS ONE. 2013;8(2):e57914. doi:.

40. Vouking MZ, Enoka P, Tamo CV, Tadenfok C. N.Prevalence of intestinal parasites among HIV patients at the Yaoundé Central Hospital, Cameroon. Pan African Medical Journal. 2014;18:136. DOI:.

41. Missaye A, Dagnew M, Alemu A. Prevalence of intestinal parasites and associated risk factors among HIV/AIDS patients with pre-ART and on-ART attending Dessie Hospital ART clinic, Northeast Ethiopia. AIDS Res Therapy. 2013;10:7.

42. Tian LG, Wang TP, Lv S, Wang FF, Guo J, Yin XM. HIV and intestinal parasite co-infections among a Chinese population: an immunological profile. IDP. 2013;2:18.

43. Bartelt LA, Dillingham R, Sevilleja JE, Barrett LJ, Guerrant RL, Bessong PO. High Cryptosporidium parvum anti-IgG seroprevalance among HIV-positive adults in Limpopo and other regions of South Africa. Am J Trop Med Hyg. 2011;85:235-6.

44. Nsagha G. Prevalence and Predisposing Factors to Intestinal Parasitic Infections in HIV/AIDS Patients in Fako Division of Cameroon. BMJ Journal. 2017;2:A41. 
45. Kwenti TE, Nkume FA, Tanjeko AT, Kwenti TDB. The Effect of Intestinal Parasitic Infection on the Clinical Outcome of Malaria in Coinfected Children in Cameroon. PLoS Negl Trop Dis. 2016;10(4):e0004673. doi:.

46. Angora KE, Djohan V, Konaté A, Kiki-Barro PC, Bedia-Tanoh AV. Uncomplicated Malaria and Intestinal Helminth Co-Infections among Schoolchildren in Abobo District, Abidjan (Côte d'Ivoire). J Infect Dis Epidemiol. 2019;5:064. .

47. Sumbele IUN, Sama SO, Kimbi HK, Taiwe GS. Malaria, moderate to severe anaemia, and malarial anaemia in children at presentation to hospital in the Mount Cameroon area: across-sectional study. Anemia. 2016;2016:5725634. doi:.

48. Teh RN, Sumbele IUN, Meduke DN, Ojong ST, Kimbi HK. Malaria parasitaemia, anaemia and malnutrition in children less than 15 years residing in different altitudes along the slope of Mount Cameroon: prevalence, intensity and risk factors. Malar J. 2018;17:336. .

49. Assefa M, Abegaz WE, Belay M. Prevalence and correlates of anemia among HIV infected patients on highly active anti-retroviral therapy at Zewditu Memorial Hospital, Ethiopia. BMC Hematol. 2015;15:6.

50. Ako SE, Njunda LA, Akum EA, Benjamin PT, Assob JC. Hematological Related Disorders and Transfusion of HIV Patients on Highly Active Antiretroviral Therapy (HAART) in the South West Region of Cameroon: Hematological Monitory Parameters for HIV Follow-Up. J HIV Retrovirus.2018;4;1:5.

51. Lai JL, Chen YH, Liu YM, Yuan JJ, Lin J, Huang AQ, Ye HH. Prevalence and risk factors of anaemia in hospitalised HIV-infected patients in southeast China: a retrospective study. Epidemiol Infect. 2019;147:e81.

52. Gebremedhin KB, Haye TB. Factors Associated with Anemia among People Living with HIV/AIDS Taking ART in Ethiopia. Hindawi Advances in Hematology. 2019; 8 .

53. Ageru TA, Koyra MM, Gidebo KD, Abiso TL. Anemia and its associated factors among adult people living with human immunodeficiency virus at Wolaita Sodo University teaching referral hospital. PLoS ONE. 2019;14(10) .

54. Volberding PA, Levine AM, Dieterich D, Mildvan D, Mitsuyasu R. Saag M for the Anemia in HIV Working Group. Anemia in HIV Infection: Clinical impact and evidence-based management strategies. Clin Infect Dis. 2004;38:1454-63.

55. Blake M, Sherer R. Management of the adverse effects of antiretroviral therapy and medication adherence. Clin Infect Dis. 2000;30(Suppl2):96-116. .

56. Ojurongbe O, Oyeniran O, Alli O, Taiwo S, Ojurongbe T, Olowe A, Opaleye O, Adeyeba O. Prevalence of Plasmodium falciparum Parasitaemia and its correlation with haematological parameters among HIV-Positive individuals in Nigeria. J Trop Med Hyg. 2014:Article ID 161284. .

57. Breymann C. Iron deficiency anemia in pregnancy. Semin Hematol. 2015;52(4):339-47.

58. Tay SC, Badu K, Mensah AA, Gbedema SY. The prevalence of malaria among HIV seropositive individuals and the impact of the co- infection on their hemoglobin levels. Ann Clin Microbiol Antimicrob. 2015;14:10. . 
59. NjuaYafi C, Achidi EA, AnchangKimbi JK, Apinjoh TO, Mugri RN, Chi HF, Tata RB, Njumkeng C, Nkock EN, NkuoAkenjl T. Malaria, helminths, coinfection and anaemia in a cohort of children from Mutengene, south western Cameroon. Malar J. 2016;15:69. DOI .

60. Enawgaw B, Alem M, Addis Z, Melku M. Determination of hematological and immunological parameters among HIV positive patients taking highly Active Antiretroviral treatment and treatment naïve in the Antiretroviral therapy clinic of Gondar University Hospital, Gondar, Northwest Ethiopia: A comparative cross-sectional study. BMC Hematol. 2014;14:8.

61. Akinbami A, Oshinaike O, Adeyemo T, Adediran A, Dosunmu O. Hematologic Abnormalities in treatment-naïve HIV patients. Infect Dis Res Treat. 2010;3:45-9.

62. Carbonara S, Fiorentino G, Serio G. Response of severe HIV associated thrombocytopenia to highly active antiretroviral therapy including protease inhibitors. J Infect. 2001;42:251-6.

63. Araga o PM, Marques HH. Trombocitopenia associada ao HIV. J Bras Aids. 2005;6(4):165-88.

\section{Figures}

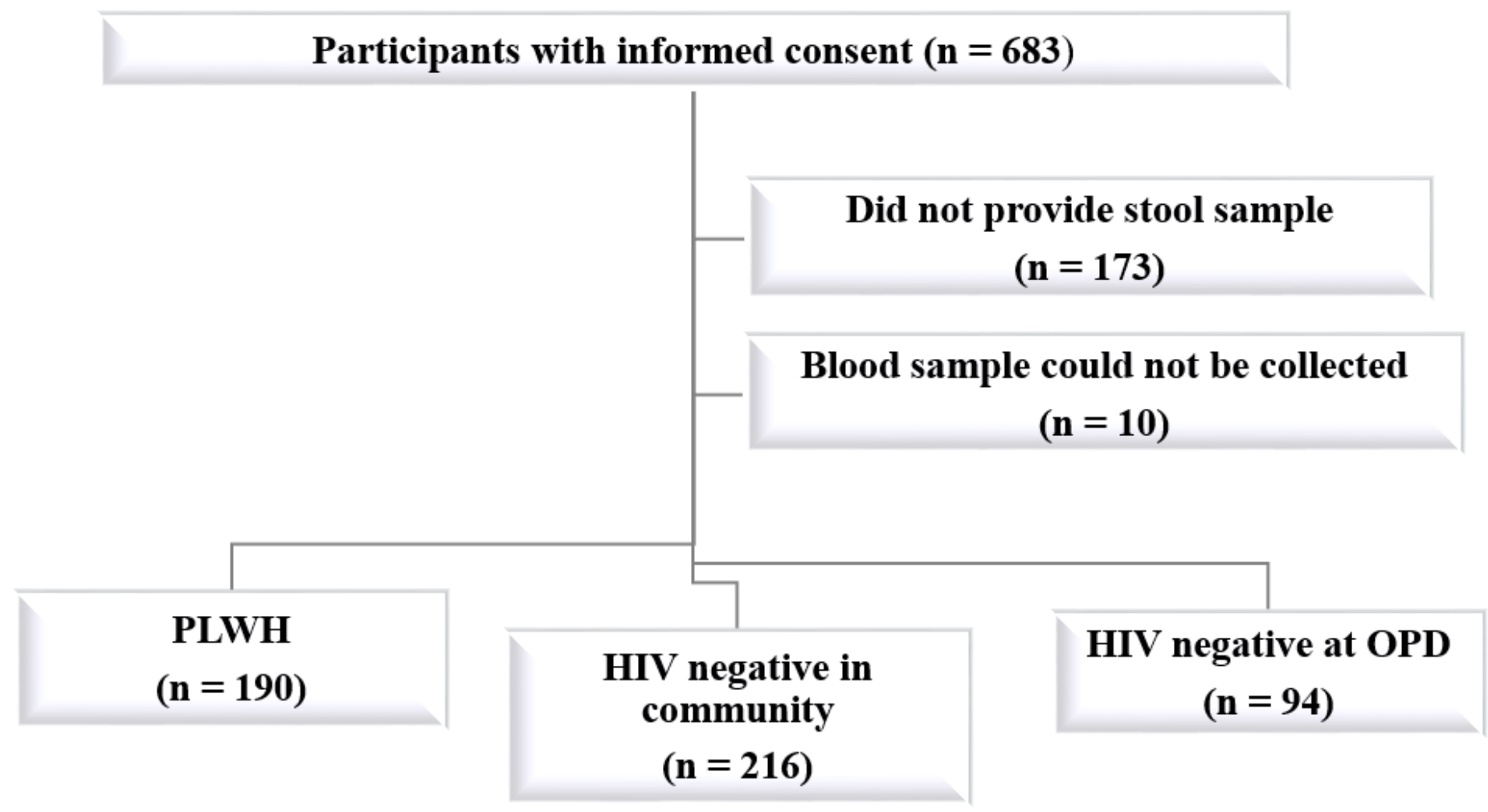

Figure 1

Study flow diagram showing compliance of the participants. 


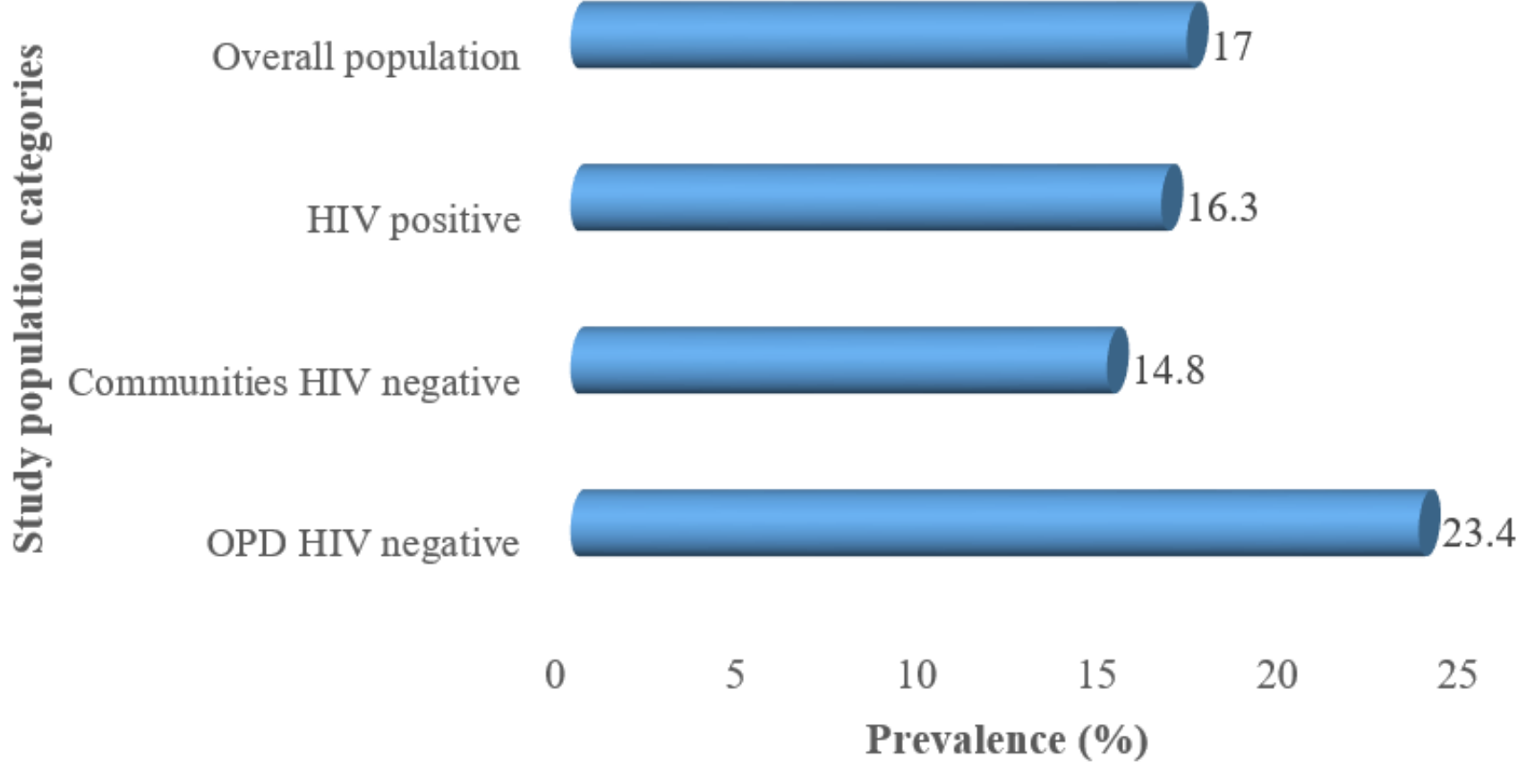

Malaria parasite prevalence

Figure 2

Prevalence of malaria parasites (MP) among the different study groups 


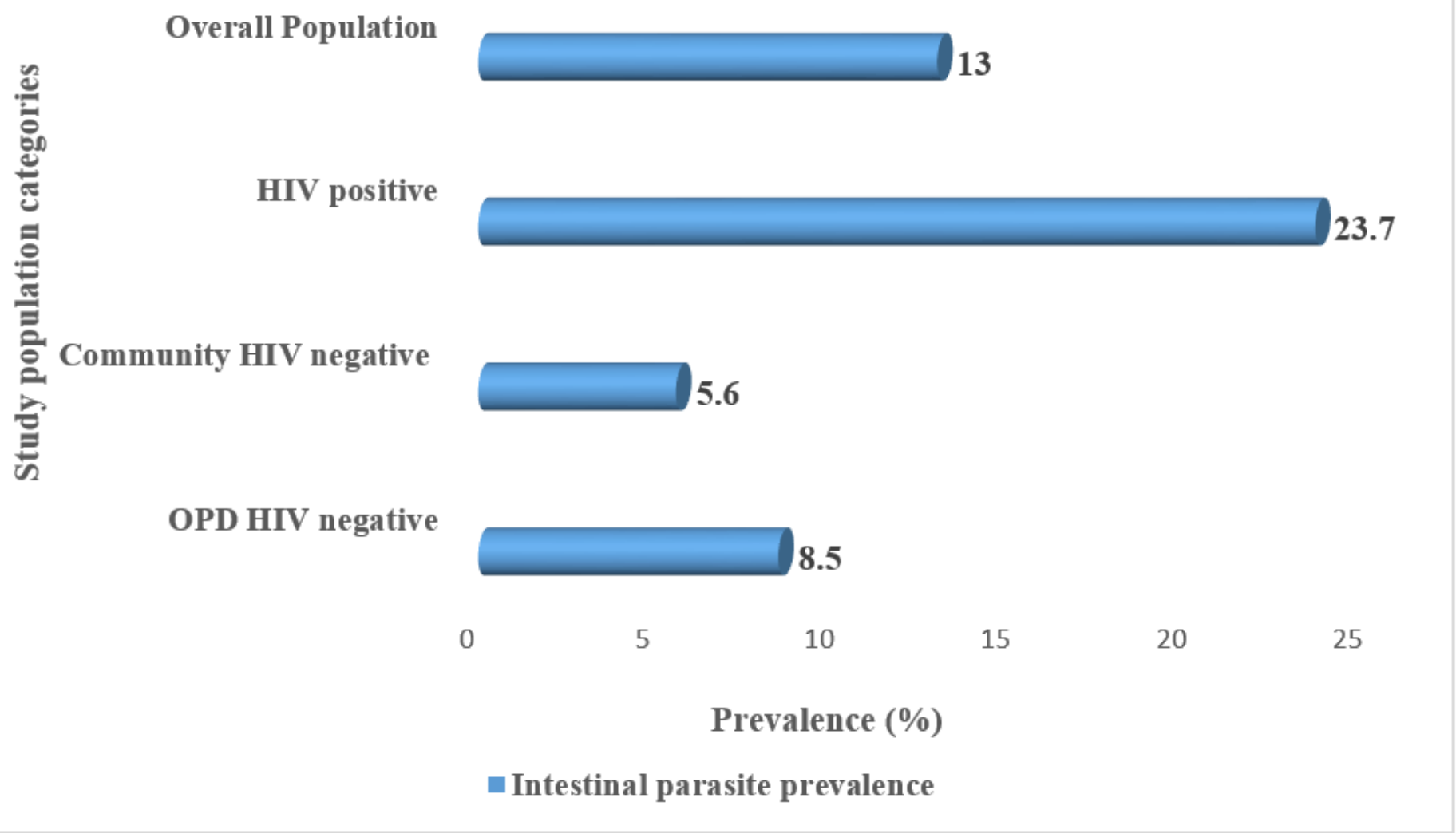

\section{Figure 3}

Prevalence of intestinal parasites (IPs) in the different study groups

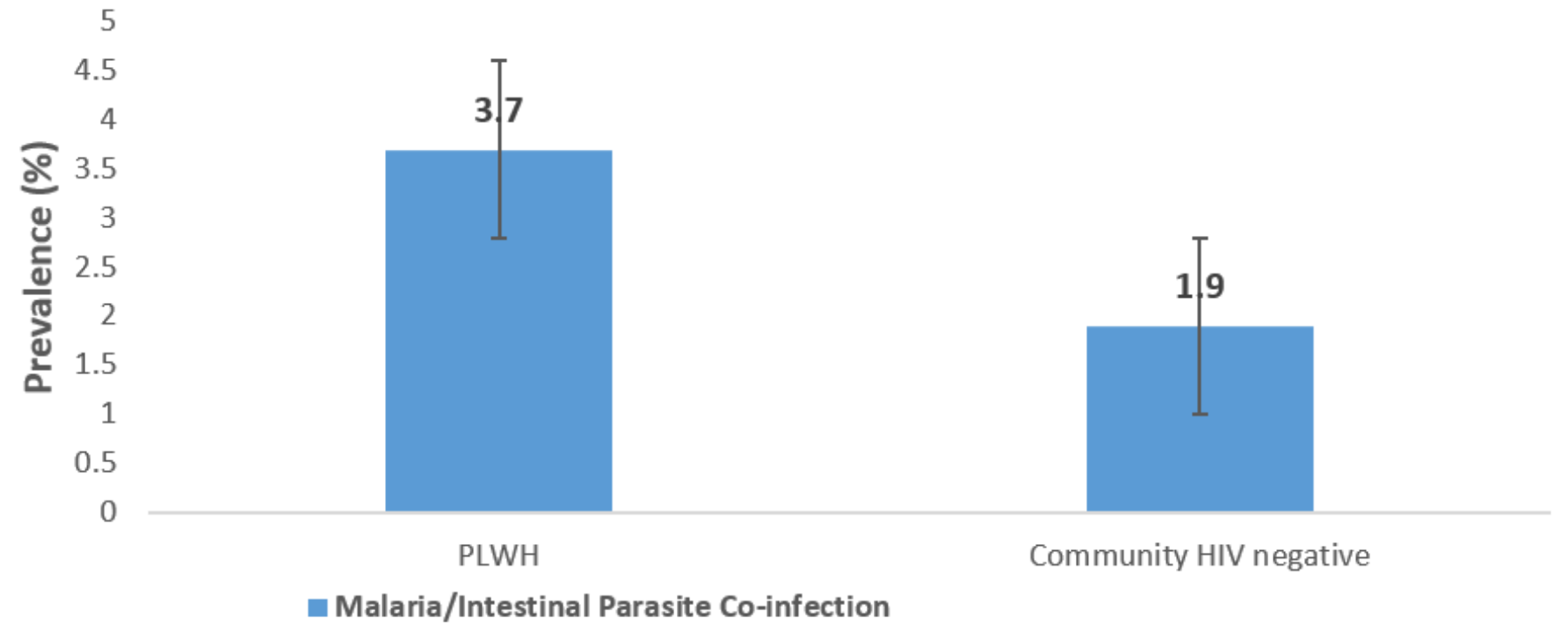

Figure 4 
Prevalence of MP/IPs co-infection in the study population

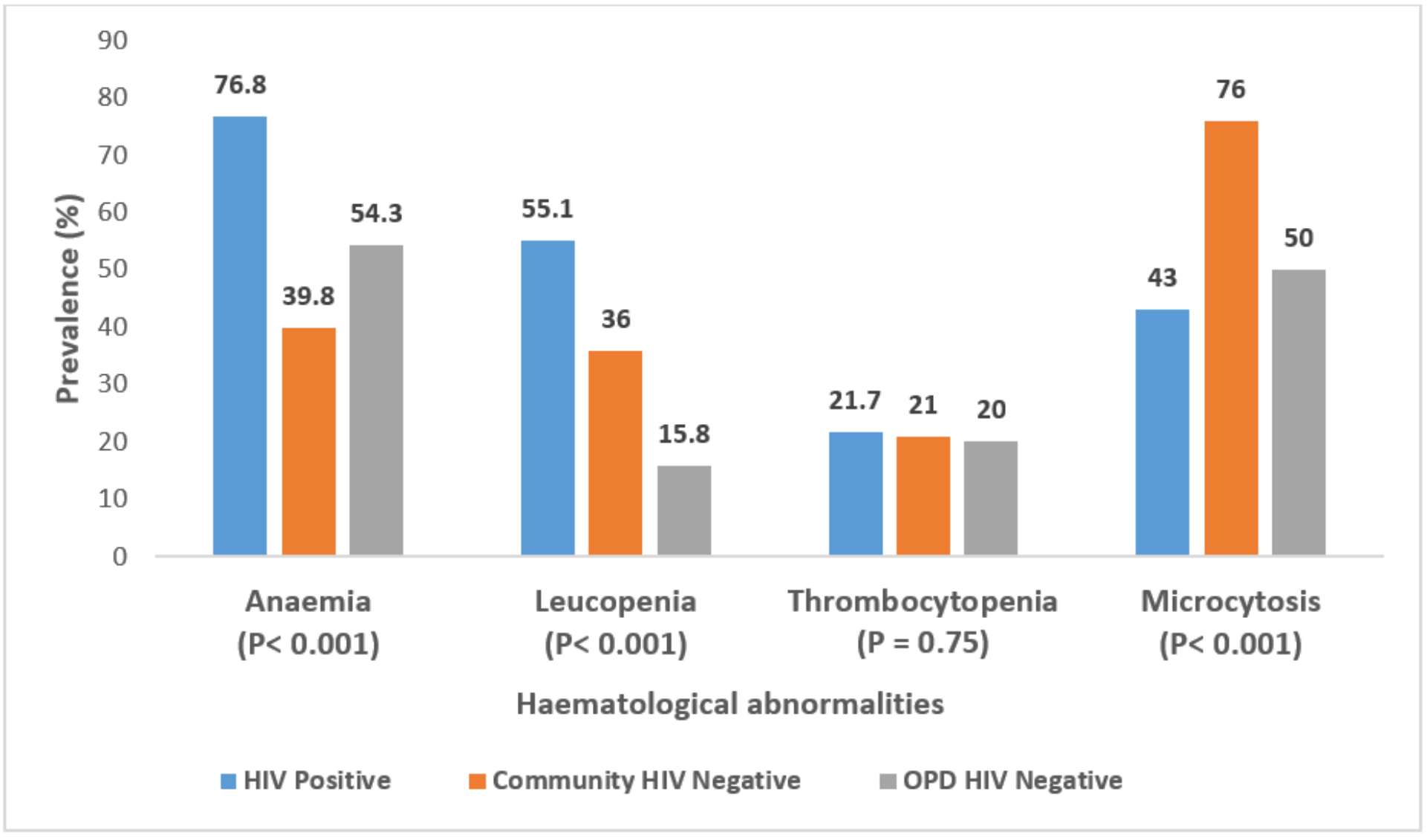

Figure 5

Prevalence of haematological abnormalities in the study population 


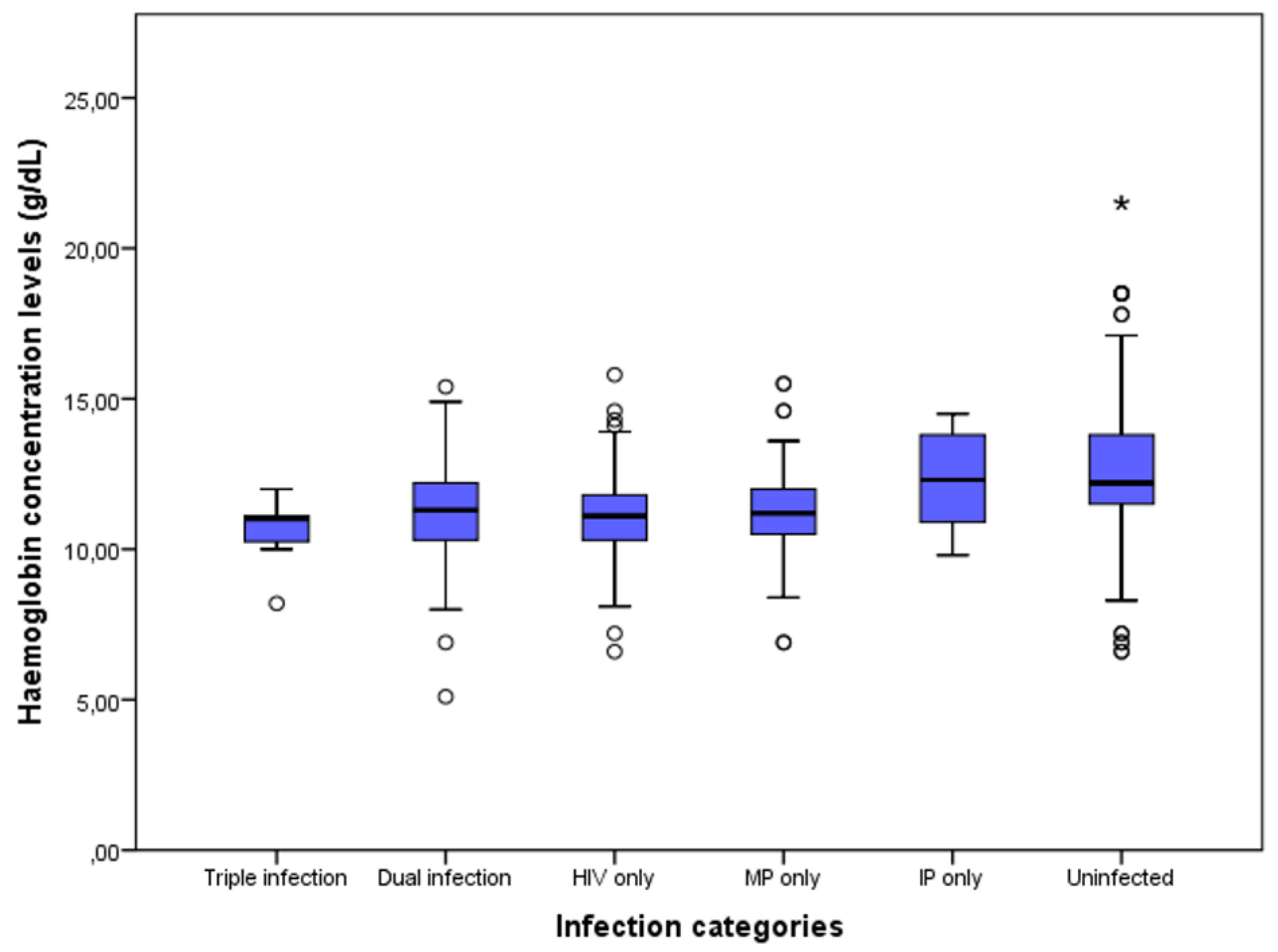

Triple infection: Participants infected with HIV, malaria and intestinal parasites.

Dual infection: Participants infected with any two (HIV/malaria, HIV/IPs, MP/IPs).

Figure 6

Box and whisker plots showing mean haemoglobin levels by infection categories. 